\title{
Conception Pyramid Method for Cultural Product Form Development
}

\author{
Tsai-Lin Yang and Ming-Chyuan Ho \\ Graduate School of Design \\ National Yunlin University of Science and Technology, Taiwan \\ philina@stu.edu.tw, homc@yuntech.edu.tw
}

\begin{abstract}
Existing methods are found incapable of meeting the needs in form designs of cultural product development and representation, particularly in the aspects of cultural identity and spiritual implication. Focusing on cultural products and with an emphasis on decorative forms, this study proposed the "Gestalt Layer Construct", based on the concepts of text, context and gestalt, among others. Accordingly, a "Conception Pyramid Method for Cultural Product Form Development" is introduced, which consists of six elements, namely Determining subject and collecting creative content, Selecting and allocating idea components, Developing gestalt concept and building gestalt layers, Identifying the best gestalt context, Defining storylines and interpreting gestalt concept, and Implementing and integrating form components. Through a series of metalwork design practices, the proposed procedures were validated and found that it features with innovativeness, usefulness, transferability and effectiveness.
\end{abstract}

Keywords: Cultural Product, Form Development, Design Method, Gestalt Layer, Metalwork Practice.

\section{$1 \quad$ Introduction}

Designing cultural products is different from designing the traditional, functional, and/or high-tech products, as it requires more emphasis on spiritual content, experiences and emotions. In this case, "Form Follows Functions" or function-oriented design methods are no longer applicable. Form workers have been influenced by the so called "creative industries trends" and unconsciously continued passing on the culture through traditional moral messages (for example, bats represent wealth and bottles imply safety in Chinese cultural context) to create contemporary art. As for cultural product design, a design method that can directly fully interpret cultural allusions or descriptions needs to be developed. Generally, product form is divided into functional and decorative forms (Luh, 1996). The functional form emphasizes functions and rational perceptions that relate to a system and logic of the science and technology, while the decorative form places an emphasis up on culture and emotions in affective and spiritual contexts in terms of human thoughts and knowledge related to philosophy. Cultural entrepreneurs focus on stories for marketing strategies; in the meantime, design trends have evolved from function-driven to user-oriented, which 
have gradually become a user experience-oriented or user interpretative approach. The importance of rational product functions has gradually declined, while the focus on affective approach applied on cultural perceptions has significantly increased. The main purpose of this study is to propose a conceptual decorative form design method for developing cultural products that not only represent but also reinvent cultural contents.

\section{Gestalt Layer Construct}

This research applies text as the main body, metaphor, and Gestalt contexts based on free creative interpretation from the designers/artists and users/audiences in order to propose a method called the "Gestalt Layer Construct" (Figure 1) for developing form design within certain creative contexts. There are three main components in this approach, namely the idea component, the idea sequence, and the Gestalt layer. The idea component is located at the bottom (e.g., A, B, C, etc.). The contents of which can be derived from the text, such as fictions, poetries, stories, or even images, colors, apparel, photography, music, architecture, and so on. The selection methods include various rational and analytical design research approaches and can also be based on the designer's emotions and intuitions. This research focuses on the emotional and intuitional method through which designers can freely choose all particular contents from the text that touch them the most and can create resonance within the audience as their main idea components.

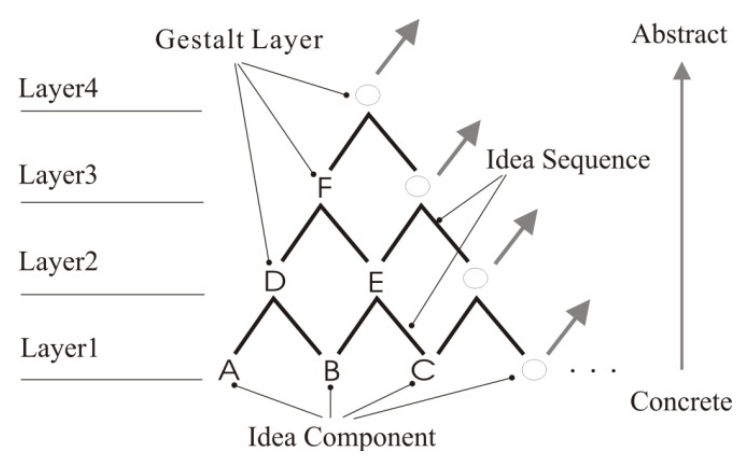

Fig. 1. The Gestalt Layer Construct

With attribute-oriented associations of the idea components, Gestalt of form is hierarchically built by creative elements from the bottom to the upper layers (e.g., D is above A and B, and for instance, "the first love" is above "orange" and "lemon" for their attributes in taste - sweet and sour). Due to its relativity, the lower layer can be regarded as the idea components of the higher Gestalt layer (e.g., D and E under F). The link between idea components is called the idea sequence, which functions to associate ideas of different layers for rich conception. The path shows the common attributes or associated meanings shared by each idea component. Each Gestalt layer can be equally regarded as creative concepts generated through the idea sequence. 
Spatial concepts therefore exist in idea components, idea sequences and Gestalt layers, while designers' imaginations, creativity, and knowledge about the text and audiences' insights will determine the height of and the amount of branches on the Gestalt Layer Construct (GLC). GLC can explicitly and systematically display how designers or artists develop their train of thought. In this way, it is possible to reveal the mystery of emotional artistic process and present it in a rational system to lead artists to higher levels of creativity and to produce various creative concepts from different perspectives and contents. Based on the aforementioned GLC framework and by expanding it into 3 dimensions, as it can be interpreted from various aspects, a Conception Pyrimid method for cultural product form development is proposed, which has six components, namely: (1) Determining subject and collecting creative content, (2) Selecting and allocating idea components, (3) Developing gestalt concept and building gestalt layers, (4) Identifying the best gestalt context, (5) Defining storylines and interpreting gestalt concept, and (6) Implementing and integrating form components.

\section{Method Demonstration}

The proposed design method will be explained based on a metalwork series entitled "Dream Weaver in the Red Chamber", a series of metal artwork by Yang (2003) based on a Chinese literature classic entitled The Dream of the Red Chamber. The full series of metal artwork is a set of accessories symbolizing the twelve maidens mentioned in the story. The artwork chosen in this study is called "Tears of Pearls," which is inspired by the fictitious character Dai-Yue Lin and based on the following reasons: (1) The Dream of the Red Chamber is passed from generation to generation and well known to almost every Chinese; (2) Each character in this fiction had distinct personalities and features; (3) Dai-Yue's personality was unique and most well known, therefore the artwork would be easily understood without redundant explanation; (4) the accessories' designs mainly focus on decorative form therefore they will not be affected by any functional issues; (5) the final results of the metal artwork will provide the most realistic interpretation of the idea components. Based on this design case and the design method proposed in this research, the measures taken to create the "Tears of Pearl" are as follows:

1. Determining subject and collecting creative content: Theoretically, all concerned citations are needed for detailed descriptions of the original appearance, personality, talents, and characteristics of Dai-Yue Lin, the main female protagonist. Due to limited length, only a partial excerpt is shown as follows:

In Chapter 3: “... She had a pair of eyes, which possessed both cheerful and sad expression, overflowing with sentiment. Her face showed some sorrow stamped on her two dimpled cheeks. She was beautiful, but her whole frame was the prey of a hereditary disease. The tears in her eyes glistened like small specks. Her balmy breath was so gentle. She was as demure as a lovely flower reflected in the water. Her gait resembled a frail willow, agitated by the wind...." 
2. Selecting and allocating idea components: Based on the excerpts, key ideas or phrases are selected as idea components (Table 1). The number of attributes can be modified as long as it can fully express the main theme of the creative subject.

Table 1. The idea components of Dai-Yue Lin

\begin{tabular}{|c|c|}
\hline Attributes & Creative component \\
\hline Name & Jade girdle hanging in the forest. \\
\hline Talent & People would merely feel sorrow about the talent. \\
\hline Appearance & $\begin{array}{l}\text { Her movement was agitated by the breeze. } \\
\text { Demure as a lovely flower reflected in the water. } \\
\text { Her beauty exceeded Hsi-Tzu's even though she possessed ailment. }\end{array}$ \\
\hline Prediction & $\begin{array}{l}\text { Crimson Pearl Grass beside the San-Sheng Stone. } \\
\text { Returned the tears of whole life. }\end{array}$ \\
\hline Personality & $\begin{array}{l}\text { The glistening tears. } \\
\text { Her heart had one more aperture than Pi-Kan's. }\end{array}$ \\
\hline
\end{tabular}

3. Developing gestalt concept and building gestalt layers: Through the semantic relationship among idea components and the artist's imaginative skill, the Gestalt layers are stacked and built into an idea tree or conception pyramid (Figure 2).

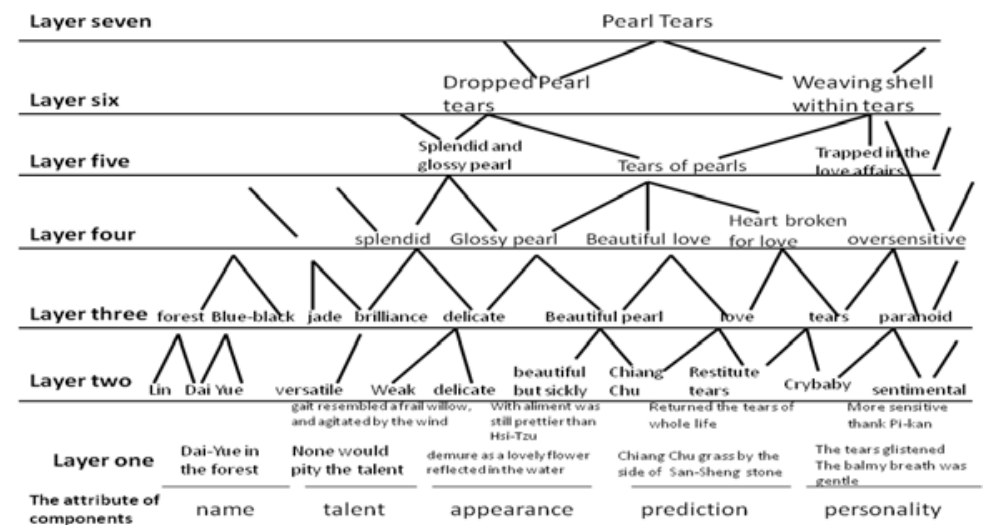

Fig. 2. The construction of an idea tree or conception pyramid

4. Identifying the best gestalt context: Gestalt-oriented concepts should be liberated from standard thinking and include lower layers of idea components (Figure 3). The best Gestalt-oriented layer is chosen from top to bottom, and it has the following qualities: coverage of most attractive attributes, easy interpretation in Gestalt title (for instance, "pearl" is easier than "beauty" for association by artists and users), and depth in layers (the deeper the better, for more attractive story and text implications). 


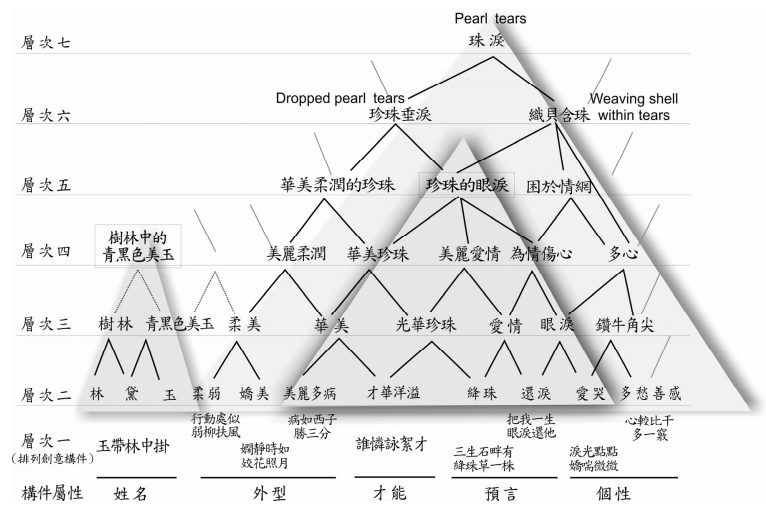

Fig. 3. Selection of a Gestalt-Oriented Concept and its Conception Pyramid

5. Defining storylines and interpreting gestalt concept: Based on the best Gestaltoriented concept of 'tears of pearls' and exclusion of minor components, it is possible to define the form element of each component. The contents and forms covered by the Gestalt-oriented concept can be concretely expressed in images, such as 'pearls', 'love', 'tears', 'paranoid' (in Chinese, it literally means ox horn), etc. Based on the concrete form of each component, the artist should choose one or several idea sequences through which to define the main story line. (Figure 4) The artist then searches for forms of each component derived from the three main story lines that possess strong imagery. Through various comparisons, the shell shapes derived from 'weaving shell within tears' possessed stronger visual representations that could be selected as the main elements of the visual design. On the other hand, tears of pearls, cones, web of love, and heart shapes can also be used as complementary elements. Other elements such as flower petals representing 'delicate', which are not closely related to the story line, were eliminated because they conflicted with the shell shape.

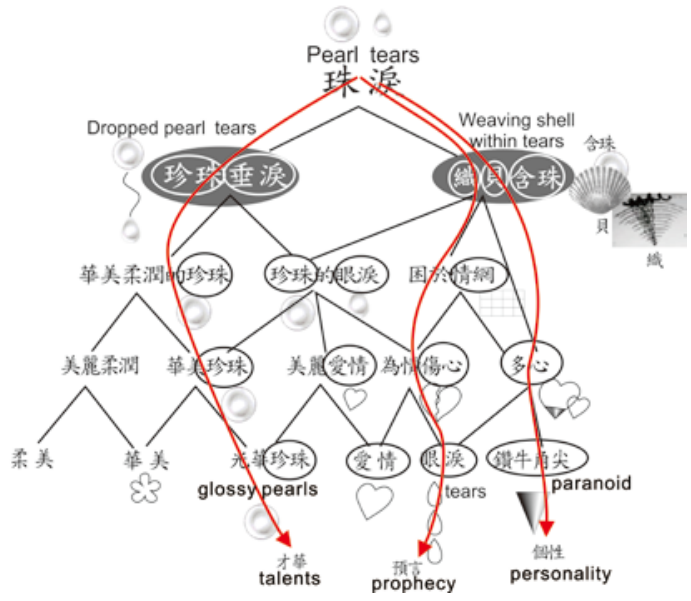

Fig. 4. Concretization of form components and definition of the story line 
6. Implementing and integrating form components: Based on the visual elements derived from 'weaving shell within tears', the glossy pearl is in the middle of the shell-shaped woven metal and represents Dai-Yue's talent, while the metal weaving symbolizes the web of love; the weaving technique was implemented to interpret how Dai-Yue is always being 'trapped in the web of love'. Furthermore, the shape combination of heart and cone represents the 'paranoid' characteristics. 'Tears of pearls' applied the shape of tears with pearls as the tears. Based on the pearl inside the shell and the cone holding the shell facing downwards like tears, this design expressed that Dai-Yue Lin was a crybaby and had a paranoid personality. (Figure 5)

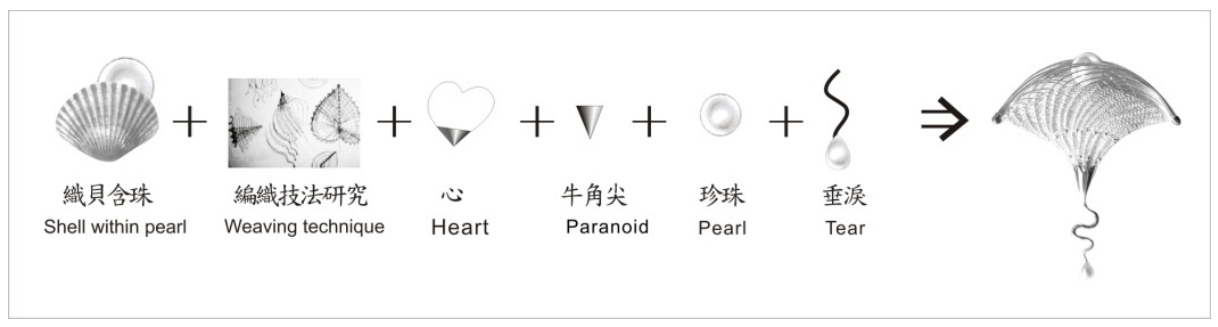

Fig. 5. Main visual elements of one of the final designs

The series of jewelry design generated by the implementation of the form creation method proposed in this research consist of five components-a necklace, a hairpin, a brooch, and a pair of earrings. The design was modified to fit the functional requirements and the final work was entitled "Tears of Pearls" (Figure 6).
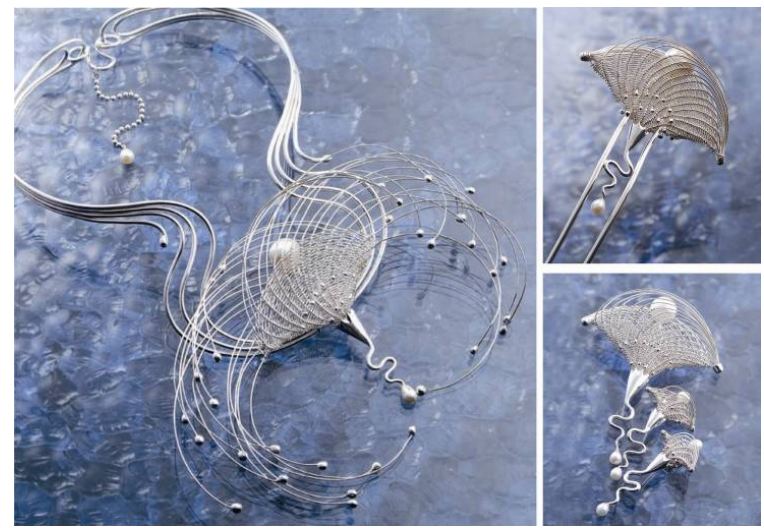

Fig. 6. A series of artworks that interpret Dai-Yue Lin's characteristics

\section{$4 \quad$ Verification}

The proposed process was implemented on the "Dream Weaver of the Red Chamber," and generated 12 series of jewelry design concepts that successfully interpret each 
role's primary Gestalt-oriented context and develop original and innovative (or significantly differing from general thinking) jewelry designs for females. The suggested method possessed the feasibility and practicability to be implemented in the real design process to develop a new metal weaving technique. These 12 series of metal artworks (each consists of five to eight components) were shown to an expert on The Dream of the Red Chamber, who has been the former dean of the College of Liberal Arts at the National Cheng Kung University, Taiwan. He was asked to name each character based on these jewelry series. Amongst the twelve series of metal artworks, except for Pao-Chai Hsue's controversial characteristics, he successfully identified five main characters. The other works were also recognized after hints were provided. Based on verification by the expert, this method was proven valid.

Afterwards, a one-month public exhibition was held for these twelve series of metal artworks. Based on the feedback provided by the audiences, people who had certain knowledge about the twelve maidens in The Dream of the Red Chamber would stay longer to appreciate the artwork and were able to guess the main characters without any hints. "Tears of Pearls" was also submitted to the 55 $5^{\text {th }}$ Taiwan Province Art Exhibition and was awarded the First Prize (there were 112 pieces of metalwork submitted in total and 33 pieces were selected for the final round). Another six of the twelve series of metal artwork were submitted to various national level design competitions and successfully won a total of six awards. Based on the performance of the final design works, the validity of this design method was proven. For professional artists in the same field, especially those who participate in national competitions, it is known that the discernment of technical skills is low. Therefore, the main consideration of the judges is creative expressions in terms of main themes and form creations. From this viewpoint, the design method proposed in this research contributed references for this particular subject matter. Furthermore, this method was also taught in a formal teaching curriculum to guide students in the conduction of design activities in order to understand the transferability of the design method proposed in this research. The student design projects conducted based on this design method have successfully achieved various design awards, for examples: Silver Prizes of the 2008 and of the 2010 Young Designers' Exhibition, First Prize of the 2011 Creative Uniform Design Competition, and First Place Award of the 2012 Swarovski Jewelry Design Competition. Based on students' responses and performances, it is assured that this design method possesses transferability and learnability.

\section{Conclusion}

This research introduced the Conception Pyramid Method for cultural product form development. Through a series of metalwork design practices, the suggested procedures were validated and found that it features with innovativeness, usefulness, transferability and effectiveness. The new approach has five characteristics:

1. The process of selecting idea components is based on an existing text, therefore the cultural elements extracted from the text relatively would be more objective and the selection and construction of idea components would also be more holistic and 
unbiased. On the other hand, as the admirers possess certain knowledge on the text, they can also observe the design results with more objective and holistic views.

2. The development of the Gestalt-oriented concept depends on personal experience, cultural contents, education, customers, and many other factors. For the same text, it is possible to generate different kinds of Gestalt-oriented concepts and structures that will create diverse representations and lead to various possible art directions.

3. Through different combinations of idea sequences, artists can be lead to develop original ideas with rich creative contents. At the same time, it is possible for artists to keep their creative thinking on track and transform it into a rationally comprehensive and systematical framework.

4. In creative art, it is possible to project personal emotions. In the same series of artwork, the artist can generate different artwork based on his/her personal experience at different times and keep developing new ideas.

5. It is possible to conduct form creation or design in a team. Based on different cultural backgrounds and life experiences, the artists can work together and generate a richer creative capacity.

In the field of creative design and art, researchers need to further explore the rationale of design processes that lead to final design results. The results of design and art creations often have the characteristics of a Gestalt-oriented concept and are difficult to be separated into pieces for verification. Apart from the text, other symbols such as graphics, images, movement, sound, and numbers that can facilitate artists to communicate and apply their ideas can also be researched further.

Acknowledgments. This work was partially supported by the National Science Council (NSC101-2622-E-366-001-CC3) of Taiwan.

\section{References}

1. Cao, X., Shih, H.: The Story of the Stone. The first bookstore, Tainan (1983) (verified)

2. Luh, D.B.: Influential Factors and Forces Analysis on Stylability Development. Journal of Design 1(1), 33-50 (1996)

3. Yang, T.L.: Weaving Dream of the Stone history - Reinventing Ornaments in Classic Chinese Literature through Semantic Approach. Unpublished Master's Thesis. National Tainan University of Arts, Tainan (2003) 